\title{
Platelets are associated with xenograft tumor growth and the clinical malignancy of ovarian cancer through an angiogenesis-dependent mechanism
}

\author{
LEI YUAN and XISHI LIU
}

\author{
Department of Gynecology, Obstetrics and Gynecology Hospital, Fudan University, Shanghai 200011, P.R. China
}

Received March 26, 2014; Accepted November 5, 2014

DOI: $10.3892 / \mathrm{mmr} .2014 .3082$

\begin{abstract}
Platelets are known to facilitate tumor metastasis and thrombocytosis has been associated with an adverse prognosis in ovarian cancer. However, the role of platelets in primary tumour growth remains to be elucidated. The present study demonstrated that the expression levels of various markers in platelets, endothelial adherence and angiogenesis, including, platelet glycoprotein IIb (CD41), platelet endothelial cell adhesion molecule 1 (CD31), vascular endothelial growth factor (VEGF), lysyl oxidase, focal adhesion kinase and breast cancer anti-estrogen resistance 1, were expressed at higher levels in patients with malignant carcinoma, compared with those with borderline cystadenoma and cystadenoma. In addition, the endothelial markers CD31 and VEGF were found to colocalize with the platelet marker CD41 in the malignant samples. Since mice transplanted with human ovarian cancer cells (SKOV3) demonstrated elevated tumor size and decreased survival rate when treated with thrombin or thrombopoietin (TPO), the platelets appeared to promote primary tumor growth. Depleting platelets using antibodies or by pretreating the cancer cells with hirudin significantly attenuated the transplanted tumor growth. The platelets contributed to late, but not early stages of tumor
\end{abstract}

Correspondence to: Mr. Xishi Liu, Department of Gynecology, Obstetrics and Gynecology Hospital, Fudan University, 419 Fangxie Road, Shanghai 200011, P.R. China

E-mail: 1xsdoc@hotmail.com

Abbreviations: CD31, platelet endothelial cell adhesion molecule 1; CD41, platelet glycoprotein IIb; TPO, thrombopoietin; IHC, immunohistochemistry; FAK, focal adhesion kinase; LOX, lysyl oxidase; BCAR1, breast cancer anti-estrogen resistance 1; VEGF, vascular endothelial growth factor; SC, serous cystadenoma; BSC, borderline serous cystadenoma; SCC, serous cystadenocarcinoma; MC, mucinous cystadenoma; BMC, borderline mucinous cystadenoma; MCC, mucinous cystadenocarcinoma; CCC, clear cell carcinoma; MVD, microvascular density; FIGO, international federation of gynecology and obstetrics; EMT, epithelial-mesenchymal transition

Key words: platelets, xenograft tumor growth, ovarian cancer, angiogenesis-dependent mechanism proliferation, as mice treated with platelet-depleting antibody 1 day prior to and 11 days after tumor transplantation had the same tumor volumes. By contrast, tumor size in the early TPO-injected group was increased significantly compared with the late TPO-injected group. These findings suggested that the interplay between platelets and angiogenesis may contribute to ovarian cancer growth. Therefore, platelets and their associated signaling and adhesive molecules may represent potential therapeutic targets for ovarian cancer.

\section{Introduction}

Ovarian cancer is one of the most life-threatening types of epithelial cancer and patients often experience rapid relapse following chemotherapy. Abnormal thrombosis and accompanied thrombocytosis can occur in ovarian cancer patients and it has been suggested that these two factors are involved in cancer metastasis. Platelets promote metastasis by protecting tumor cells from destruction by immune cells (1). They also enhance tumor cell adhesion through a selectin-GPIIb-dependent mechanism. Deficiencies in the expression of P-selectin by platelets may prevent cancer cell rolling and their adhesion to endothelial cells under flow conditions, thereby reducing the number of metastatic lesions (2). A previous study suggested that platelets mediate the epithelial-mesenchymal transition (EMT) of cancer cells (3), as platelet-derived transforming growth factor- $\beta$ (TGF $\beta$ ) and direct platelet-tumor cell contact activated the TGF $\beta /$ Smad and NF- $\mathrm{NB}$ pathways. This induced the transition of tumor cells into an invasive mesenchymal-like phenotype and enhanced tumor metastasis in vivo.

Although the role of platelets in cancer migration is well understood, the details of how platelets are involved in cancer proliferation remain to be elucidated. There is evidence indicating that platelets cause cell cycle arrest in cancer cells, however, thrombocytosis occurs in $10-50 \%$ of patients with solid malignancies (4). This has been labeled as 'reactive' or 'secondary', implying that the tumor, even small tumors, can stimulate platelet production (5). In addition, thromboipoetin (TPO), which drives thrombopoiesis, is often used to regenerate the platelet counts in patients following chemotherapy, a treatment that has been associated with tumor homing (6). Therefore, further clarification of the association between platelets and tumor proliferation may assist in elucidating the mechanisms underlying carcinogenesis and is of clinical importance (7). 
In the present study, the correlation between thrombogenic markers and malignancies of ovarian tumor was investigated. The expression of coagulant factors from patients with benign, borderline and malignant phenotypes were determined and human tumor tissue samples were stained using antibodies against platelet glycoprotein IIb (CD41), a marker for platelets, and other biomarkers associated with angiogenesis, including platelet endothelial cell adhesion molecule 1 (CD31), vascular endothelial growth factor (VEGF), lisyl oxidase (LOX), focal adhesion kinase (FAK) and breast cancer anti-estrogen resistance 1 (BCAR1). The roles of platelets in ovarian cancer growth were also determined in a transplanted mouse model. Tumor growth patterns, animal survival rate, platelet-depletion and infusion in the murine tumor model were assessed. The results provided evidence that platelets contribute to the proliferation of ovarian cancer by mediating angiogenesis, which may be important for the development of intervention strategies to treat ovarian cancer.

\section{Materials and methods}

Patients and specimens. A total of 140 patients with histologically confirmed ovarian tumors, including serous cystadenoma (SC), borderline serous cystadenoma (BSC), serous cystadenocarcinoma (SC), mucinous cystadenoma (MC), borderline mucinous cystadenoma (BMC), mucinous cystadenocarcinoma (MCC) and clear cell carcinoma (CCC), were recruited by the Obstetrics and Gynecology Hospital of Fudan University between 2008 and 2010. Each histotype contained 20 tissue specimens. The patients all underwent primary surgery at the the Obstetrics and Gynecology Hospital of Fudan University for pelvic masses. Surgery was advised if the diameter of the pelvic mass exceeded $5 \mathrm{~cm}$, and patients with physioloigal ovarian cysts were excluded. International Federation of Gynecological Oncologists (FIGO) staging was assigned to the tumors following surgery if possible.

The patient charts were reviewed to obtain clinicopathological data regarding age, preoperative blood coagulation function (activated partial thromboplastin time, prothrombin time, international normalized ratio, fasting blood glucose and D-dimer), pre- and post-operative platelet level, preoperative serum levels of CA125, CA199, carcinoembryonic antigen and $\alpha$-fetoprotein, diagnosis, histology, FIGO staging (if any), residual disease following tumor cytoreductive surgery (if any) and overall survival rate. Tissue blocks, which were harvested at the time of surgery and subsequently formalin fixed, paraffin embedded and archived in the pathology department of the hospital in the dark at room temperature, were retrieved for further immunohistochemical (IHC) staining.

Additional fresh tissue specimens from 12 patients (six with malignant epithelial ovarian tumors and six with benign tumors) were collected following patient consent for immunofluorescent staining.

The present study was reviewed and approved by the Ethics Committee of the Obstetrics and Gynecology Hospital of Fudan University, Shanghai, China.

IHC. The tissue samples, obtained from all 140 patients with benign, borderline and malignant ovarian tumors, were retrieved and serial $4-\mathrm{mm}$ sections were obtained from each block. The first slide was stained for hematoxylin and eosin to confirm the pathological diagnosis and the subsequent slides were stained for CD31, CD 41, FAK, LOX, BCAR1 and VEGF, respectively. Routine deparaffinization and rehydration procedures were performed following online protocols (http:// www.bioworldantibodies.com/info/IHC.pdf).

For antigen retrieval, the slides were heated at $98^{\circ} \mathrm{C}$ in either an EDTA-Tris buffer ( $\mathrm{pH} \mathrm{9.0)} \mathrm{or} \mathrm{citric} \mathrm{acid} \mathrm{for} 30 \mathrm{~min}$ and cooled naturally to room temperature. EDTA-Tris buffer was used for the FAK and LOX staining, while citric acid was used for the CD31, CD41, BCAR1 and VEGF staining. Endogenous peroxidase was blocked using $3 \% \mathrm{H}_{2} \mathrm{O}_{2}$ in methanol for $10 \mathrm{~min}$ prior to washing with phosphate-buffered saline (PBS). The sections were then incubated with the following primary antibodies: rabbit anti-CD31 polyclonal antibody (1:50; ab28364), rabbit anti-CD41 polyclonal antibody (1:200; ab63983), rabbit anti-FAK polyclonal antibody (1:200; ab4803), rabbit anti-LOX polyclonal antibody (1:200; ab31238), rabbit anti-BCAR1 polyclonal antibody (1:150; ab31831) and rabbit anti-VEGF polyclonal antibody (1:200; ab46154) (Abcam, Cambridge, UK) overnight at $4^{\circ} \mathrm{C}$. The secondary antibody used in this study was Sunpoly-H II (Shanghai Sunbio Co., Shanghai, China).The slides were rinsed and incubated with horseradish peroxidase-labeled secondary anti-rabbit antibody detection reagent (Shanghai BioTech Company, Ltd., Shanghai, China) at room temperature for $30 \mathrm{~min}$. The bound antibody complexes were stained for 3-5 min, or until appropriate for microscopic examination with diaminobenzidine and then counterstained with hematoxylin (30 sec) and mounted. Negative controls were generated by incubating slides with PBS instead of the primary antibody.

The immunoreactivity staining was characterized quantitatively using a semi-quantitative scoring system, as described previously (8). The number and intensity of positive cells was quantified using Image-Pro Plus 6.0 software (Media Cybernetics, Inc., Rockville MD, USA) in a blinded-manner. A series of five random images on several sections were captured to obtain a mean value.

The microvascular density (MVD) of the containing CD31-stained slides was assessed using light microscopy (Eclipse Ni-U; Nikon Corp., Tokyo, Japan) in the areas having the highest number of capillaries and small venules (neovascular hot spots). Subsequently, the microvessels were counted on 10 selected (x200) fields of the 'hot plot' by the same investigator in a blinded-manner. The MVD was defined as the mean vessel count obtained in these fields, as reported by Ma et al (9).

Immunofluorescent staining. The freshly harvested tissues were formaldehyde-fixed prior to freezing and were sectioned using a cryostat (Leika CM1850; Leica Microsystems GmbH, Nussloch, Germany) to $4 \mu \mathrm{m}$. The tissue sections were then incubated with rabbit anti-CD41 polyclonal antibody (1:100; ab63983), mouse anti-CD31 monoclonal antibody (1:100; ab24590) and/or mouse anti-VEGF monoclonal antibody (1:200; ab1316), which were all purchased from Abcam, overnight at $4^{\circ} \mathrm{C}$. The tissue sections were then incubated with a secondary goat anti-rabbit fluorescein isothiocyanate (111-095-003; Jackson ImmunoResearch Laboratories, Inc., West Grove, PA, USA) and goat anti-mouse Rhodamine (115-295-003; Jackson ImmunoResearch Laboratories, Inc.) at $37^{\circ} \mathrm{C}$ for $1 \mathrm{~h}$. The nuclei were counterstained with diamidinophenylindole (Sigma-Aldrich, St. Louis, MO, 
USA) and fluorescent images were captured using microscopy (BX51; Olympus, Tokyo, Japan) and analyzed using Image-Pro Plus software for colocalization.

Tumor growth and survival rates in the mouse model. To assess the effects of different treatments on the tumor growth and mouse survival rates, SKOV3 human ovarian cancer xenografts in athymic nude mice were used. The animals were purchased from Biomodel (Shanghai Research Center for Model Organisms), and were kept under standard conditions of $25^{\circ} \mathrm{C}$, 40-60\% humidity and a 12-h light/dark cycle, and access water and food ad libitum. Tumor loci were generated by injecting $4 \times 10^{6}$ tumor cells subcutaneously into 6-8-week-old female mice (weight, $18-20 \mathrm{~g}$ ) on day 0.

For the thrombin and hirudin treatment groups, prior to tumor transplantation, the ovarian tumor cells were pretreated with thrombin, an activator of platelets $\left(1 \mathrm{U} / 10^{6}\right.$ cells; Sigma-Aldrich) or hirudin, a specific inhibitor of thrombin $\left(0.1 \mu \mathrm{g} / 10^{6}\right.$ cells) donated by Professor Houyan Song, Fudan University (Shanghai, China) for 10 mins. The other groups were treated 7 days after tumor transplantation with either: Platelet depletion, involving administration of rat anti-mouse GPIb immunoglobulin ( Ig)G antibody $(2 \mu \mathrm{g} / \mathrm{g}$, Emfret Analytics, Eibelstadt, Germany) via the tail veins once every 5 days; Isotype control, involving administration of rat IgG (2 $\mu \mathrm{g} / \mathrm{g}$, Emfret Analytics) via the tail veins once every 5 days; platelet infusion, in which the platelets first separated from the platelet-rich plasma of the blood by centrifugation, washed by gel filtration and resuspended in buffer containing $137 \mathrm{~mm} \mathrm{NaCl}, 4 \mathrm{~mm} \mathrm{KCl}, 0.5 \mathrm{~mm} \mathrm{MgCl}, 0.5 \mathrm{~mm}$ $\mathrm{Na}_{2} \mathrm{HPO}_{4}, 11.1 \mathrm{~mm}$ dextrose, $0.1 \%$ bovine serum albumin and $10 \mathrm{~mm} \mathrm{~N}$-2-hydroxyethylpiperazine- $N$ '-2-ethanesulfonic acid ( $\mathrm{pH} 7.4$ ), as previously described (10). Briefly, in the platelet infusion group, platelets were first separated from the platelet rich plasma (PRP) of blood by centrifugation at $280 \mathrm{xg}$ for $8 \mathrm{~min}$, the plasma and buffy coat were gently transferred to a fresh tube and the centrifugation was repeated at $280 \mathrm{xg}$ for $4 \mathrm{~min}$. Platelets were isolated by filtering the resulting PRP through a Sepharose 2B (Sigma-Aldrich) column equilibrated with Pipes buffer (25 mM Pipes, $137 \mathrm{mM} \mathrm{NaCl,} 4 \mathrm{mM}$ $\mathrm{KCl}, 0.1 \%$ dextrose; $\mathrm{pH} 7.0$ ) and then resuspended in buffer containing $137 \mathrm{mM} \mathrm{NaCl}, 4 \mathrm{mM} \mathrm{KCl}, 0.5 \mathrm{mM} \mathrm{MgCl}_{2}, 0.5 \mathrm{mM}$ $\mathrm{Na}_{2} \mathrm{HPO}_{4}, 11.1 \mathrm{mM}$ dextrose, $0.1 \%$ bovine serum albumin, $10 \mathrm{mM} N$-2-hydroxyethylpiperazine- $N$ '-2-ethanesulfonic acid ( $\mathrm{pH} 7.4)$. The platelets $\left(6 \times 10^{8}\right)$ from two donor mice in buffer were then injected into a single recipient xenograft mouse via the tail vein and platelet infusion was repeated every 5 days until experimental termination or TPO, involving administration of recombinant human (rh)TPO $(50 \mathrm{U} / \mathrm{mouse} / \mathrm{day}$; 3Sbio, Inc., Shenyang, China) for 7 days.

The tumor volume and the number of mice remaining alive following tumor cell xenograft (lethalities were due to dyscrasia of the tumor growth) were measured on days 7, 12, 17, 22, 27, 32 and 37. The volumes of the in situ tumors were defined as $\mathrm{V}=\pi \mathrm{ab}^{2} / 6$ (a, major diameter; b, minor diameter) (11) and measured using calipers.

Effect of platelets on early developing and fast growing periods in vivo. Based on previous in vitro studies demonstrating the effects of platelets on the promotion of primary ovarian tumor growth (12), the present study evaluated the role of platelets in tumor growth during different growing periods of carcinogenesis (early developing, vs. fast growing periods).

In the early platelet depletion group (E-PD/E-non-immune), the mice received either rat anti-mouse GPIb or control rat IgG antibodies via the tail vein 1 day prior to subcutaneous inoculation of the tumors (-1 day). The antibody regimes were repeated every 5 days until experimental termination. In the late platelet depletion group (L-PD/L-non-immune), the mice received either rat anti-mouse GPIb or control rat IgG via the tail vein 11 days post-tumor inoculation, which was repeated every 5 days until experiment termination.

In the early TPO treatment group (E-TPO), from -4 day, the mice were injected subcutaneously with rhTPO for 4 days. The mice then received injection once every 4 days (rhTPO injection on days 1 and 5). For the late-TPO treatment group (L-TPO), starting from day 11, the mice received with TPO for 4 days (between days 11 and 14). The purpose of starting the TPO injections at -4 days for the E-TPO group was to enable the platelet counts to rise 2-fold above that of the control at day 0 . This treatment enabled the platelet count to be comparable to that of platelet infusion.

Each of the E-TPO, E-PD, E-non-immune, L-TPO, L-PD and L-non-immune groups were comprised of 8 mice. For all treatment groups, the mice were sacrificed by cervial dislocation on day 21. The volumes of the in situ tumors were defined as $V=\pi \mathrm{ab}^{2} / 6$, measured using calipers.

Statistical analysis. For descriptive statistics, box plots were used to graphically depict groups of immunoreactivity data and clinical parameters. The bottom and top of the box represent the lower and upper quartile, respectively, the band close to the middle of the box represents the median and open circles indicate outliers (values $>1.5 \mathrm{x}$ interquartile range). Pearson's or Spearman's rank correlation coefficient were used to evaluate the correlations between the variables when the two variables were continuous or when at least one variable was ordinal. The association between various clinical and pathological parameters was compared using $\chi^{2}$ tests. The present study compared continuous variables using Student's t-test or analysis of variance (ANOVA) and used non-parametric tests (Mann-Whitney), when appropriate, to compare differences. $\mathrm{P}<0.05$ was considered to indicate a statistically significant difference. All statistical analyses were performed using SPSS 16.0 software (SPSS, Inc., Chicago, IL, USA).

\section{Results}

Patients with a poor prognosis are prone to thrombophilia. To compare the differential expression levels of coagulation factors and molecular markers among a variety of human pathological conditions, clinical specimens from 140 patients with SC, BSC, SCC, MC, BMC, MCC and CCC were used for analysis of coagulation indices and IHC markers (each histotype had 20 specimens, respectively). No differences in ages were apparent among the clinical categories (mean=46.1; standard deviation=17.8). The malignant histotypes (SCC, MC and CCC) had a higher D-dimer level, a marker for fibrin generation (Fig. 1A) and a shorter thrombin clotting time compared with the benign 

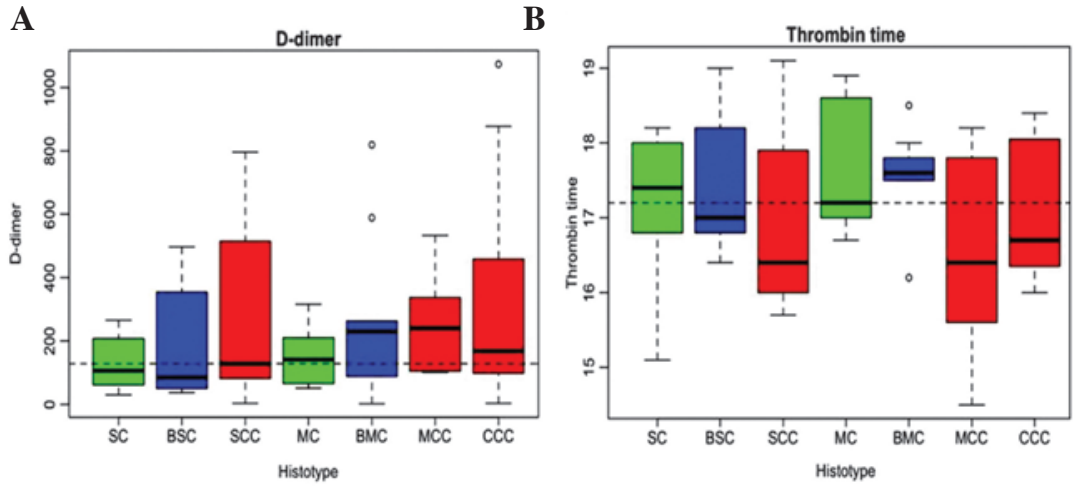

Figure 1. (A) Malignant histotypes (SCC, MC and CCC) had higher levels of D-dimer (marker for fibrin generation) and (B) shorter thrombin clotting time scompared with the benign samples. The coagulation indices of clinical specimens from 140 patients with SC, BSC, SCC, MC, BMC, MCC, CCC were analyzed. No differences in ages were observed among clinical categories (mean=46.1, standard deviation=17.8). Bars represent the maximum and minimum values, open circle indicate outliers. SC, serous cystadenoma; BSC, borderline serous cystadenoma; SCC, serous cystadenocarcinoma; MC, mucinous cystadenoma; BMC, borderline mucinous cystadenoma; MCC, mucinous cystadenocarcinoma; CCC, clear cell carcinoma.

A

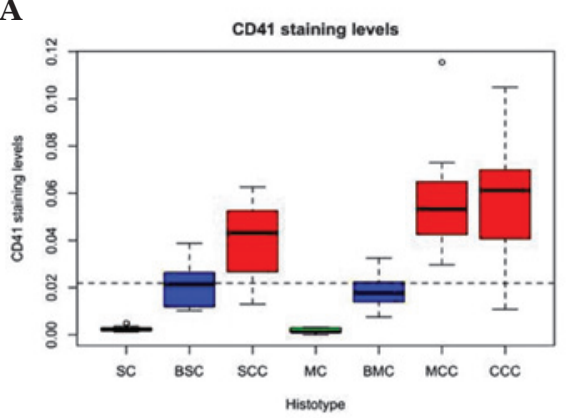

C

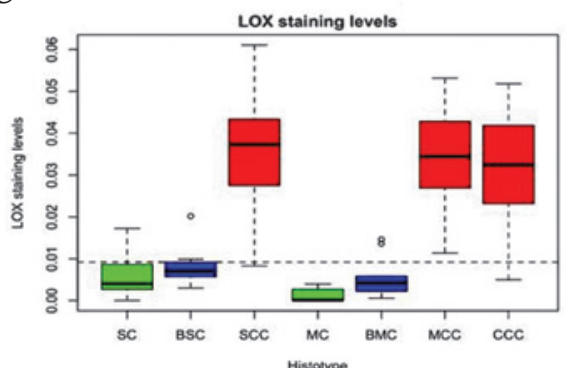

E

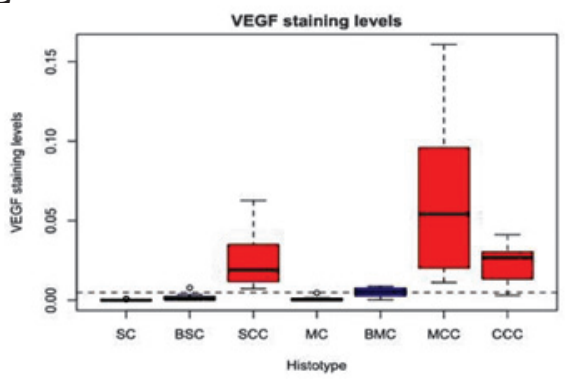

B

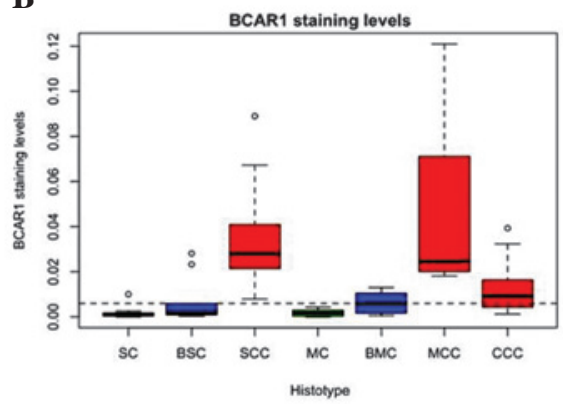

D

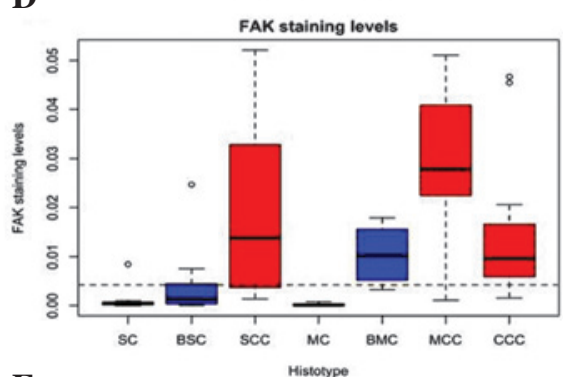

$\mathbf{F}$

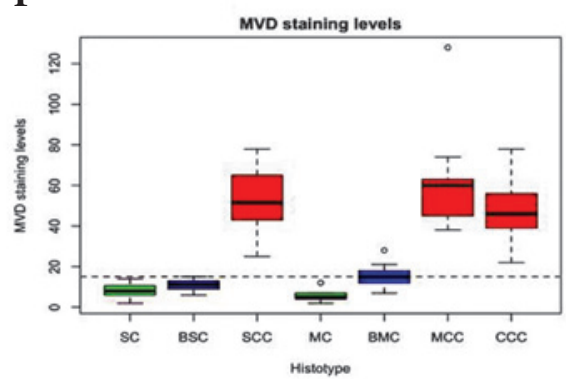

Figure 2. Differences in the expression levels of CD41, BCAR1, LOX, FAK, VEGF and CD31 (MVD) in different histoypes. (A) CD41; (B) BCAR1; (C) LOX; (D) FAK; (E VEGF and (F) MVD staining among the different histotype groups. Clinical specimens from 140 patients with SC, BSC, SCC, MC, BMC, MCC and CCC were subject to analysis of immunohistochemical markers. No differences in ages were observed among the clinical categories (mean=46.1, standard deviation=17.8). Bars represent the minimum and maximum values, open circles indicate outliers. SC, serous cystadenoma; BSC, borderline serous cystadenoma; SCC, serous cystadenocarcinoma; MC, mucinous cystadenoma; BMC, borderline mucinous cystadenoma; MCC, mucinous cystadenocarcinoma; CCC, clear cell carcinoma; LOX, lysyl oxidase; FAK, focal adhesion kinase; VEGF, vascular endothelial growth factor; MVD, microvascular density.

samples, as analyzed by ANOVA ( $\mathrm{P}<0.05$; Fig. 1B). Prior to surgery, the carcinoma patients had a significantly higher platelet count compared with either the borderline cystadenoma or cystadenoma $\left(281 \pm 103 \times 10^{9}, 262 \pm 108 \times 10^{9}, 209 \pm 41 \times 10^{9}\right.$, respectively;
$\mathrm{P}=0.03$ ). By contrast, following tumor removal surgery, the platelet counts were equal in the three groups. Notably, the preoperative platelet counts were positively correlated with the clinical stages in the malignant histotypes $(\mathrm{r}=0.494 ; \mathrm{P}=0.004)$. 
$\mathbf{A}$

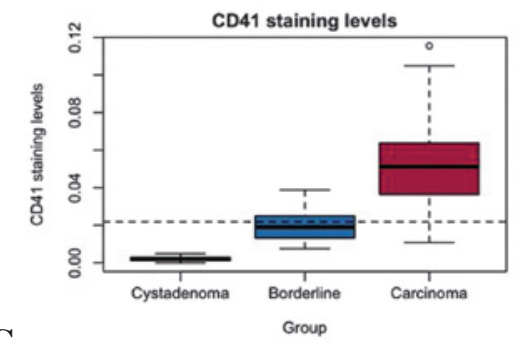

C

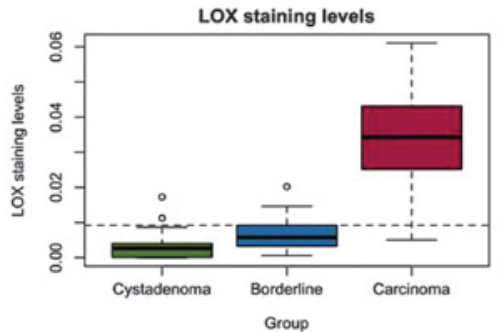

$\mathbf{E}$

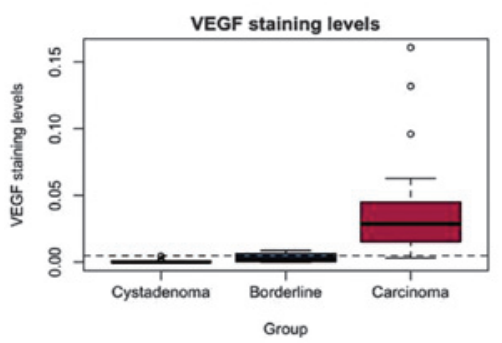

B

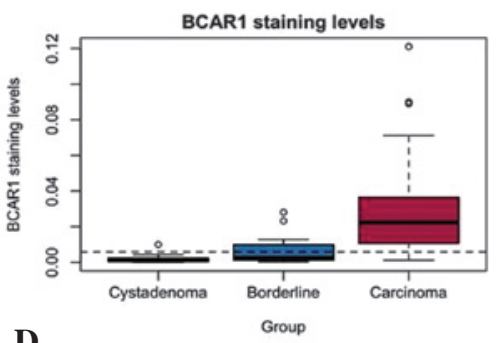

D
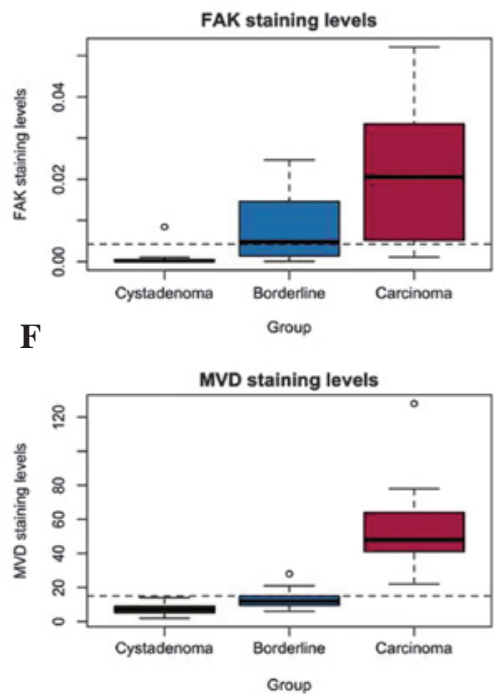

Figure 3. Expression levels of CD41, BCAR1, LOX, FAK, VEGF and MVD in patients with carcinoma, borderline cystadenoma or cystadenoma group. (A) CD41, (B) BCAR1, (C) LOX, (D) FAK, (E) VEGF and (F) MVD staining among the different histotype groups. Histotypes were regrouped into cystadenoma, borderline and carcinoma. Bars represent the minimum and maximum values, open circles indicate outliers. CD41, platelet glycoprotein IIb; BCAR1, breast cancer anti-estrogen resistance 1; LOX, lysyl oxidase; FAK, focal adhesion kinase; VEGF, vascular endothelial growth factor; MVD, microvascular density.

A

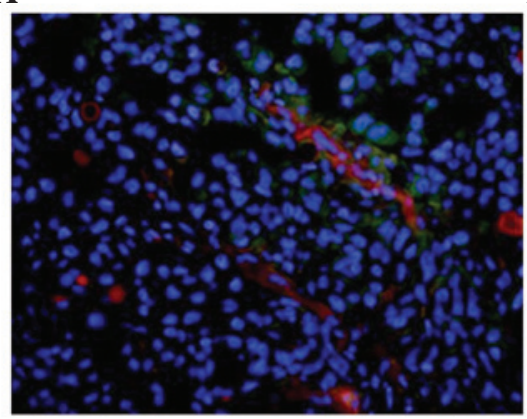

B

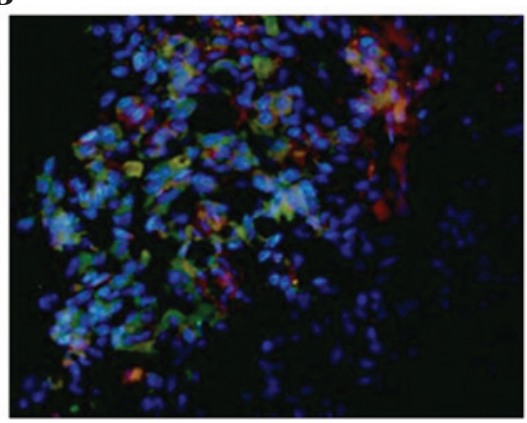

Figure 4. Colocalization of the platelet marker CD41 (green) with (A) CD31 (red) or (B) VEGF (red) in human ovarian mucinous cystadenocarcinoma. Tissues $(4 \mu \mathrm{m})$ were incubated with anti-CD41, CD31 and VEFG antibodies prior to staining with fluorescein isothiocyanate and rhodamine-conjugated secondary antibodies. The nuclei were stained using diamidinophenylindole (blue). The samples were analyzed by fluorescence microscopy (magnification, 400x). CD41, platelet glycoprotein IIb; ;CD31, platelet endothelial cell adhesion molecule 1; VEGF, vascular endothelial growth factor.

To analyze the phenotypes of the cancer specimens, angiogenic, tumorigenic and thrombogenic markers, including CD41, CD31 (MVD), BCAR1, VEGF, LOX and FAK, were specifically selected. CD41, a platelet marker and receptor (GPIIb/IIa), mediates platelet adhesion to endothelial cells and augments endothelial angiogenesis (13). Tumor cell adhesion to endothelium-bound platelets is mediated by GPIIb-IIIa in flow (14). BCAR1, also referred to as p130 Crk-associated substrate, is located at the sites of adhesion and is associated with tumor migration, colony formation and anchorage-independent growth
(15-18). FAK is responsible for the uninhibited proliferation of cancer cells, their protection from apoptosis, invasion, migration, adhesion and spreading, as well as tumor angiogenesis (19). VEGF also induces angiogenesis and tumor growth (20). There were significant differences in the expression levels of CD41, BCAR1, LOX, FAK, VEGF and CD31 (MVD) in the different histoypes ( $\mathrm{P}<0.05$; Fig. 2A-F). Regrouping of the seven histotypes into three groups: cystadenoma, borderline cystadenoma and carcinoma, according to their outcomes, revealed that patients with carcinoma expressed significantly more CD41, 
$\mathbf{A}$

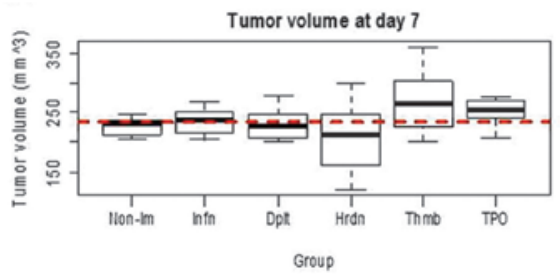

$\mathbf{C}$

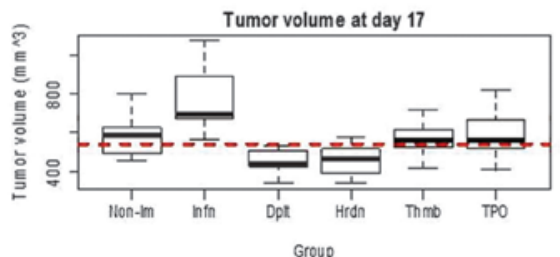

$\mathbf{E}$

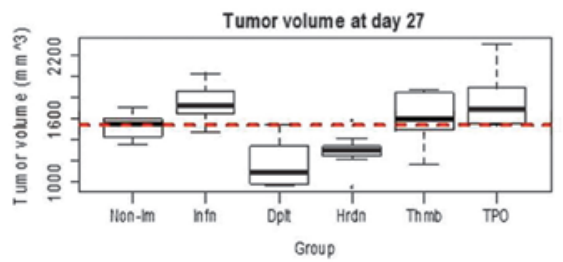

B

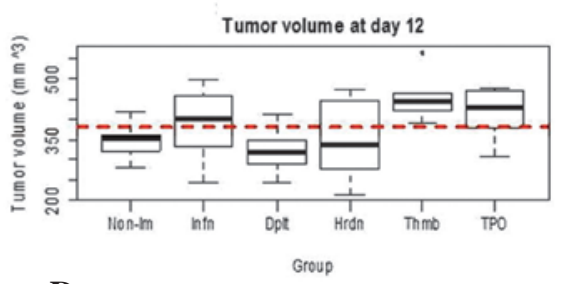

D

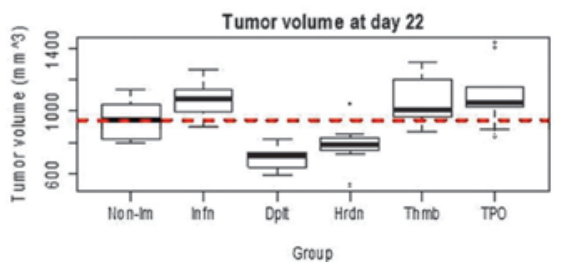

$\mathbf{F}$

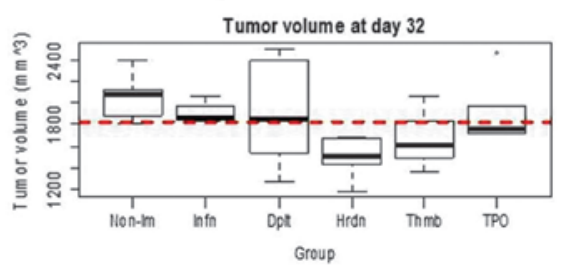

Figure 5. Effects of platelets in growth of tumor volume. Nude mice were subcutaneously inoculated with SKOV3 tumor cells and grouped according to treatment type: Non-immune group (injection of platelet non-immune antibody with $2 \mu \mathrm{g} / \mathrm{g}$ control rat IgG every 5 days); depletion group (injecting $2 \mu \mathrm{g} / \mathrm{g}$ platelet depletion rat anti-mouse GPIb IgG antibody every 5 days); infusion group (infusing platelets from two donor mice into a single recipient every 5 days); TPO group (subcutaneous injection of $50 \mathrm{U} /$ mouse/day TPO for 7 days); Thrombin group (thrombin treatment prior to subcutaneous transplantation); hirudin group (hirudin treatment prior to subcutaneous transplantation) and control group (tumor subcutaneous transplantation without any treatment). The tumor volumes $\left(\mathrm{mm}^{3}\right)$ were recorded on days (A) 7, (B) 12, (C) 17, (D) 22, (E) 27 and (F) 32. Bars represent the minimum and maximum values, open circles indicate outliers. Non-Im, non-immune group; Infn, infusion group; Dplt, depletion group; Hrdn, hirudin group; Thmb, thrombin group; TPO, thrombopoietin.

BCAR1, LOX, FAK, VEGF and MVD compared with the borderline or cystadenoma groups (Fig. 3A-F). In addition, the expression levels of CD41, CD31 (MVD), BCAR1, VEGF, LOX and FAK were positively correlated with the clinical stages in the carcinoma (SCC, MC and CCC) samples $(\mathrm{r}=0.528,0.613$, $0.575,0.575,0.43,0.571$ and 0.589 , respectively). The expression levels were significantly higher in patients diagnosed with late stage cancer compared to those with early stage cancer $(\mathrm{P}<0.05)$. Notably, the serum CA-125 levels were positively correlated with the expression levels of CD41 and MVD (data not shown).

In the carcinoma samples, the expression of CD41 overlapped with that of CD31 and VEGF (Fig. 4A and B), suggesting that the platelets may contribute to the generation of carcinoma by inducing angiogenesis. Furthermore, platelets formed aggregates surrounding the tumor.

Effects of platelets and thrombosis on cancer and survival rates in a xenograft mouse model. During several stages of tumor development, tumor cells acquire different capacities to sustain proliferation, evade growth suppressors and activate invasion (21). The interactions between tumors and platelets in blood vessels are critical for tumor attachment and transendothelial migration. To examine the role of platelets and coagulation in tumor development in situ, nude mice were subcutaneously transplanted with SKOV3 ovarian tumor cells prior to further treatments, including platelet depletion, platelet infusion, TPO injection and thrombin and hirudin pretreatment. The depletion of platelets with the injection of anti-GPIb IgG resulted in a decrease in tumor volume compared with the injection of

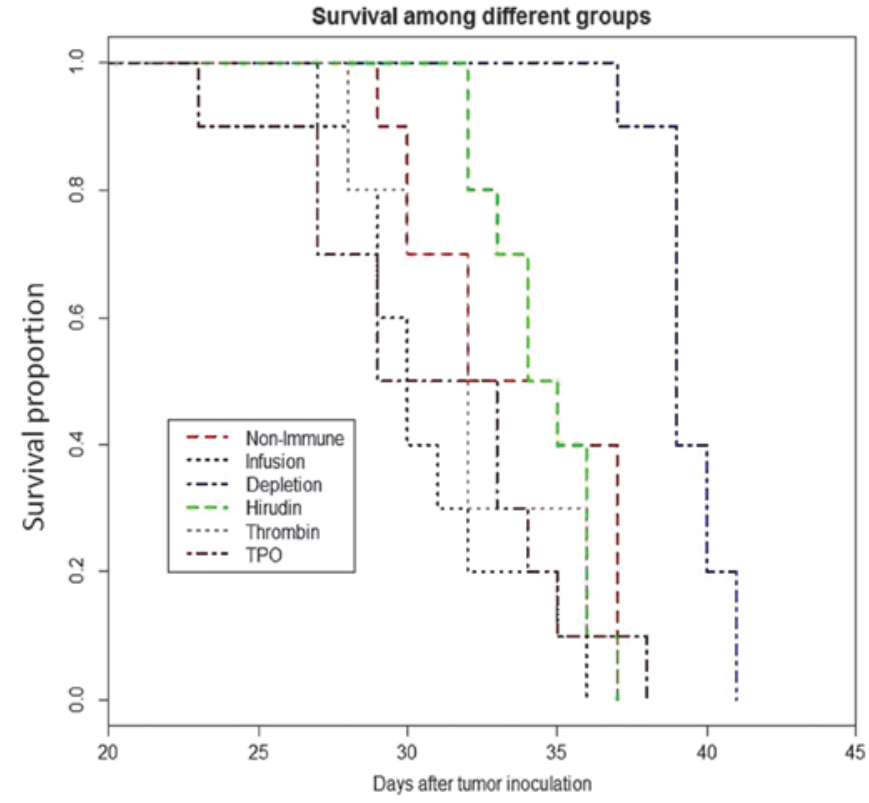

Figure 6. Effect of platelets on mouse survival rates. Nude mice were subcutaneously inoculated with SKOV3 tumor cells and grouped according to different treatments. Non-immune group (injection of platelet non-immune antibody with $2 \mu \mathrm{g} / \mathrm{g}$ control rat IgG every 5 days); depletion group (injecting $2 \mu \mathrm{g} / \mathrm{g}$ platelet depletion rat anti-mouse GPIb IgG antibody every 5 days); infusion group (infusing platelets from two donor mice into a single recipient every 5 days); TPO group (subcutaneous injection of $50 \mathrm{U} /$ mouse/day TPO for 7 days); Thrombin group (thrombin treatment prior to subcutaneous transplantation); hirudin group (hirudin treatment prior to subcutaneous transplantation) and control group (tumor subcutaneous transplantation without any treatment). The proportion of mice remaining alive were recorded on days 7, 12, 17, 22, 27, 32 and 37. TPO, thrombopoetin; Ig, immunoglobulin 

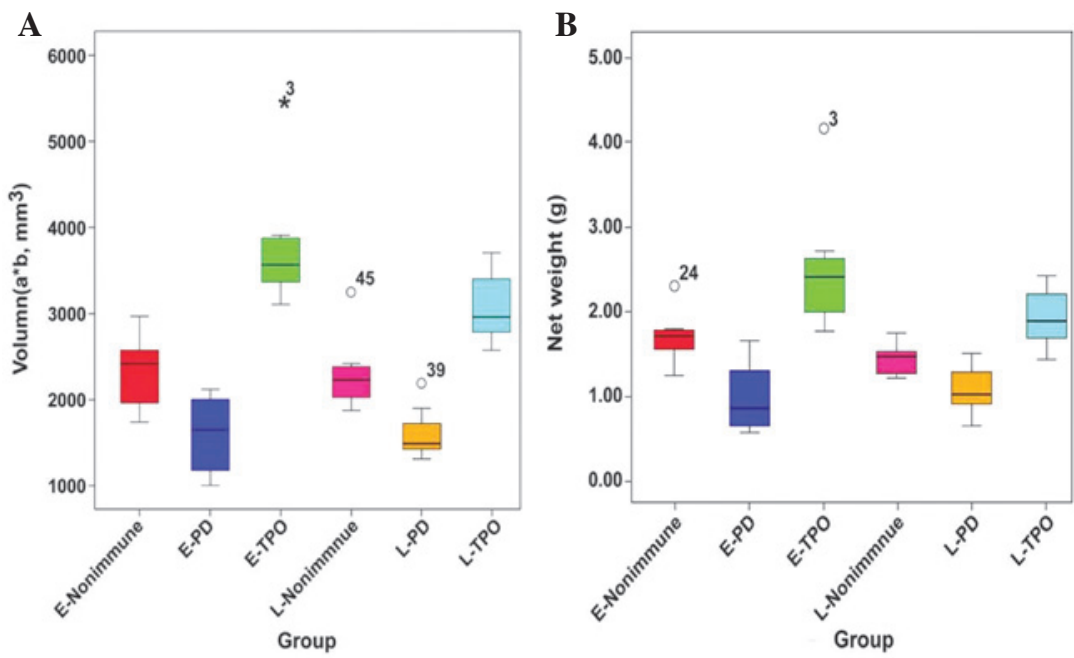

Figure 7. Effects of E-PD and L-PD (rat antimouse GPIb IgG antibody, $2 \mu \mathrm{g} / \mathrm{g}$ ): E-non-immune and L-non-immune (control rat IgG, $2 \mu \mathrm{g} / \mathrm{g}$ ) and E-TPO and L-TPO on (A) tumor volumes ( $\left(\mathrm{ab} 2 / 6, \mathrm{~mm}^{3}\right.$ ) and (B) net tumor weight $(\mathrm{g})$. $\mathrm{n}=8$ in each group. Bars represent the minimum and maximum values, open circles indicate outliers. Nonimmune, non-immune antibody injection; PD, platelet-depletion; TPO, thrombopoetin, E-, early; L-, late; Ig, immunoglobulin.
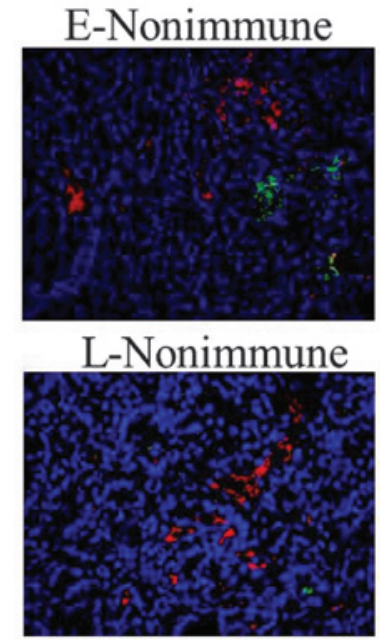

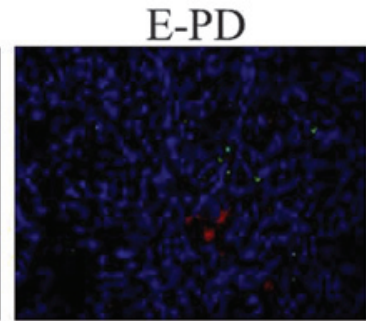

L-PD

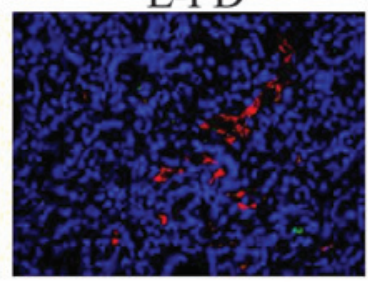

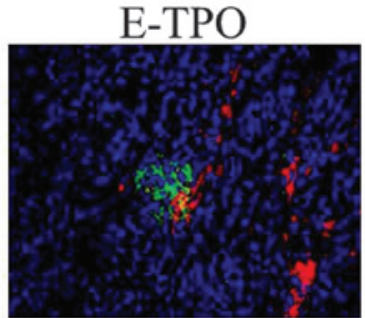

L-TPO

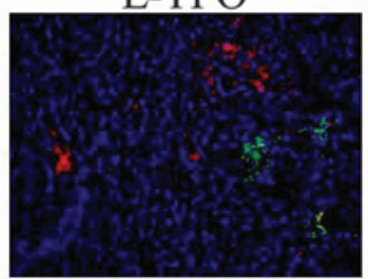

Figure 8. Effects of E-PD and L-PD and E-TPO and L-TPO treatment on the colocalization of CD41 and CD31 in tumor tissues. Images were captured under x400 magnification using a fluorescent microscope. Blue, diamidinophenylindole; green, CD41 and red, CD31. Nonimmune, non-immune antibody injection; PD, platelet-depletion; TPO, thrombopoietin; E-, early; L-, late; CD41, platelet glycoprotein IIb CD31, platelet endothelial cell adhesion molecule 1.

control non-immune antibody alone (Fig. 5). The inhibition of tumour growth by platelet depletion remained after 5 days (Fig. 5B). In addition, the effect of platelet depletion was increasingly potent with tumor progression (Fig. 5B-E). At day 27, platelet depletion reduced tumor volume by $29 \%$ compared with the control samples (Fig. 5E). However, the mice receiving platelet infusion exhibited a 1.14-fold increase in their median tumor size at day 12. The tumor-promoting effect of platelets was sustained until day 27. Notably, at day 32 the median tumor size of the platelet infusion group was comparable to that of the control (Fig. 5F), which was possibly due to the mice succumbing to mortality.

The injection of TPO, which is a factor regulating platelet production, resulted in the most profound induction of tumor growth day 12 and between days 22 and 27 (Fig. 5B-E). When the transplanted tumor was pre-incubated with hirudin, an antithrombin reagent, the tumor proliferation was reduced (Fig. 5A-E). Furthermore, the tumor-suppression effect of hirudin peaked at day 32 . However, the tumor-supressing effect of hirudin treatment was less significant than that of platelet depletion. Pretreatment of the tumor cells with thrombin, an enzyme that converts fibrinogen to fibrin, increased the tumor size compared with the non-immune antibody control (Fig. 5A-D). The difference was observed from the beginning of tumor measurement until day 27.

The effect of different treatments on the survival rate of mice bearing a human ovarian tumor are shown in Fig. 6 . The median survival rate for the non-immune antibody treated group was 33.5 days. Exogenous infusion of platelets resulted in regional transplant tumor growth and reduced the median survival rate to 30 days (Fig. 6). The median survival rate for mice receiving platelet-depleting antibody, leading to experimental thrombocytopenia, was 39 days. In addition, 9 of the 10 mice receiving the platelet-depleting antibody survived $>39$ days.

Pretreatment of the tumor cells with hirudin increased the mouse survival rates. By contrast, pretreatment with 
thrombin decreased the mouse survival rates. Notably, TPO had a dual effect on survival rate. Initially, it caused a rapid decrease in survival rate, while at the later time point, it prolonged the rates. TPO has been suggested to have additional functions to promoting platelet generation, which may affect survival rates in a non-specific manner (22).

Effects of platelets at different ovarian cancer developmental stages. To assess the roles of platelets at different cancer developmental stages (early developing and fast growing periods), two different methods of platelet depletion and TPO injection were developed, as mentioned previously. E-TPO treatment led to a significantly larger tumor volume $\left(3778.1 \pm 737.7 \mathrm{~mm}^{3}\right)$ and stripped tumor weight ( $2.48 \mathrm{~g}$ ) compared with L-TPO treatment $\left(3,075.9 \pm 405.9 \mathrm{~mm}^{3}\right.$ and $\left.1.91 \mathrm{~g}\right)$ as shown in Fig. 7A and B $(\mathrm{P}<0.05)$. By contrast, E-PD treatment resulted in smaller tumor volume $\left(1,599.0 \pm 435.9 \mathrm{~mm}^{3}\right)$ and stripped tumor weight $(0.9 \mathrm{~g})$ compared with those treated with L-PD $\left(1,660.8 \pm 294.1 \mathrm{~mm}^{3}\right.$ and $1.06 \mathrm{~g}$; Fig. 7A and B), although the difference was not statistically significant $(\mathrm{P}>0.05)$. For the control groups, tumor volume $\left(2,327.9 \pm 410.2 \mathrm{~mm}^{3}\right)$ and stripped tumor weight $(1.69 \mathrm{~g})$ following E-non-immune treatment were not significantly different compared with those of the L-non-immune treatment group $\left(2,301.5 \pm 423.7 \mathrm{~mm}^{3}\right.$ and $1.42 \mathrm{~g}$; Fig. $7 \mathrm{~A}$ and B).

Fluorescent staining revealed that TPO treatment resulted in more significant overlapping of CD41 and CD31 compared with non-immune antibody treatment (Fig. 8). Mice in the E-TPO group had a higher levels of CD41 and CD31 colocalization compared with those in the L-TPO group.

\section{Discussion}

Thrombocytosis is a notable characteristic associated with cancer progression. However, despite the fact that the metastasis-promoting effect of platelets has been investigated, the role of platelets in primary tumor proliferation remains to be full elucidated. A number of studies have suggested that platelets facilitate hematogenous metastasis $(1,2,14,23-25)$. In blood flow, tumor cells roll and firmly adhere to endothelium-bound platelets. This dynamic adhesion process is dependent on P-selectin and glycoprotein IIb $(2,14,25)$. Deficiency in these two proteins reduces the number of metastatic lesions (2). The present study demonstrated that tumour malignancy was positively correlated with the enhanced expression of CD41, CD31 (MVD), BCAR1, FAK, LOX and VEGF using IHC. By examining human ovarian cancer cells transplanted into nude mice, the present study demonstrated that platelets were relevant for tumor development and mouse survival rates. Early treatment with TPO resulted in a larger tumor loci compared with later treatment, suggesting that efficient neoplasia requires angiogenesis and platelets.

The clinical samples derived from malignant carcinoma had higher expression levels of thrombogenic and angiogenic markers, including D-dimer, based on IHC analysis. D-dimer is a degraded product of fibrin generation $(26,27)$ and its presence, which reflects the activation of the coagulation system and fibrinolysis, this has been associated with clinical tumor growth, invasion and metastasis (27). The present study revealed that malignant cancer patients had a shorter thrombin time, suggesting that the production of thrombin increased. In the tumor microenvironment, thrombin can bind to its receptor PAR-1 on the endothelium and tumor cells, inducing angiogenesis and cancer proliferation (28). Certain types of cancer, including colon carcinoma and melanoma, express tissue factors on their surface to augment local thrombogenesis. However, whether increased coagulation is the cause or the consequence of malignancy of cancer requires further investigation.

BCAR1 is one of the Crk-associated substrate protein family members and a variety of mutations in this protein confers susceptibility to ovarian cancer (16). Reduction in the expression levels of BCAR1 resulted in reduced tumor growth following docetaxel chemotherapy. BCAR1 forms a phosphorylation-dependent signaling complex with FAK and Src kinase, promoting the adhesion-mediated breast cancer survival rate (29). FAK is an important adhesion regulator, which has been suggested to promote cancer metastasis, angiogenesis and tumor progression (30). Elevated levels of FAK in serous ovarian carcinoma are associated with decreased patient survival rates (31). Studies have also suggested that VEGF-induced vascular endothelium permeability is mediated by FAK (32). The present study revealed that malignant cancer patients had increased levels of FAK, BCAR1 and VEGF. These findings implied that increased 'cross-talk' of platelets with either soluble factors or P-selectin may enhance tumor survival rate.

The expression levels of the LOX family proteins, which are secreted by tumors and have been investigated extensively, increased in various types of cancer including head and neck squamous cell carcinoma (33), breast $(33,34)$, colorectal $(35,36)$ and prostate (37) cancer. Although numerous studies have supported the roles of the LOX family members in tumor suppression and in promoting metastasis, a number failed to address the underlying mechanism. The potential contributions of LOX, BCAR1 and FAK in the malignant progression of ovarian cancer requires further investigation.

The observation that the expression pattern of VEGF overlapped with that of CD41, suggested that platelet aggregation may promote angiogenesis and tumor proliferation. The coexpression of CD41/CD31 and CD41/VEGF in tissue samples implied that platelets promoted local ovarian cancer growth through a mechanism of pro-angiogenesis. The effect of platelets on angiogenesis may be dependent on the secretion of endothelial growth factors, including platelet-derived growth factor (38). Further investigation using a platelet-tumor co-culture model are required to delineate the soluble factor expression levels.

Thrombocytosis was observed in patients with carcinomas. The cause of malignancy-associated thrombocytosis has been assumed to be attributable to interleukin-6 (IL-6) which potently promotes megakaryocyte maturation and enhances platelet production, although the cellular source of IL-6 may differ between patients (39). Using a murine tumor model, the present study demonstrated that platelet depletion or pretreatment with hirudin considerably reduced the tumor volume and increased the median mouse survival rate. These results suggested that platelets were important for tumor growth. In addition, the mice with early platelet-depletion occurred at an early stage, had a smaller tumor size compared with mice with late stage platelet-depletion. Furthermore, mice receiving early TPO injection had a larger tumor size compared with those receiving late TPO injection. However, whether the produced 
platelets affected tumor growth and angiogenesis remains to be elucidated. Levels of IL-6 are frequently increased in tumor patients and increases thrombopoiesis only in the presence of TPO, which drives thrombopoiesis. These findings are supported by the demonstration in vivo that platelets transfused into mice with orthotopic ovarian tumors enhanced the proliferation index of the tumors. TPO regulates all stages of platelet production by promoting the proliferation and maturation of megakaryocyte progenitors (22). In preclinical studies, TPO treatment resulted in a rapid increase in platelet counts, but not cytokines $(40,41)$. Truncated or full-length forms of TPO can stimulate the production of megakaryocytes and platelets in humans and enhances platelet recovery following chemotherapy and TPO may offer potential in treating thrombocytopenia in cancer patients. In the present study, TPO was administered into the tumor xenograft mice, resulting in ovarian cancer proliferation and reduced survival rates. Therefore, clinically, administration of TPO into patients with the intention of recovering platelet counts following chemotherapy may facilitate latent tumor growth and promote relapse.

Rupture of atherosclerotic plaques exposes the subendothelial collage, which then activates platelets. Activated platelets express receptors, including GPIb-IX, which is the receptor for von Willebrand factor and induces platelet aggregation (42). Platelet aggregation also requires the binding of integrin GPIIB-IIIa receptor to fibrinogen. Therefore, platelets may function as a bridge, which assists in the attachment to blood vessel walls. It has also been demonstrated that platelets could enhance the proliferation rate of human and murine ovarian cancer cells by a mechanism that requires direct cell contact (43). These findings were supported in the present study in vivo, in which platelet transfusions into mice with orthotopic ovarian tumors enhanced the tumour proliferation index.

The present study compared the effect on tumor growth of early and late platelet depletion by TPO treatment. The purpose of starting E-TPO from - 4 day was to raise platelet counts by 2 -fold on day 0 . The results indicated that tumour volume when platelet depletion occurred at an early stage of tumor formation (-1 day), were not significantly different compared with platelet depletion at an late stage of tumor formation (11 days). If platelet counts were increased by TPO, the tumor volume in early treatment samples were significantly larger compared with these in late treatment. This implied that platelets did not contribute to the early stages of tumor development, however, when tumors reached a certain size, the role of platelets in tumor proliferation was manifested. As platelets may secrete signaling/adhesive molecules, including TGF, which promote angiogenesis and initiate tumor EMT, they possibly contributed to the later stage of cancer development.

In conclusion, the present study provided in vivo evidence that platelets enhanced cell proliferation in ovarian cancer. Additionally, the clinical data suggested that malignant carcinoma cells were characteristic of platelet, angiogenesis and migratory markers. These findings suggested that thrombosis and angiogenic processes may be associated with tumor progression. The results of the present study assist in understanding the underlying mechanism of ovarian cancer growth and provide insight into new therapeutic targets for treating ovarian cancer.

\section{Acknowledgements}

The authors would like to thank Dr Sun-Wei Guo, who conceived the hypothesis of this study and assisted with the study design and data analysis.

\section{References}

1. Felding-Habermann B, Habermann R, Saldivar E and Ruggeri ZM: Role of beta3 integrins in melanoma cell adhesion to activated platelets under flow. J Biol Chem 271: 5892-5900, 1996.

2. Borsig L, Wong R, Feramisco J, et al: Heparin and cancer revisited: mechanistic connections involving platelets, $\mathrm{P}$-selectin, carcinoma mucins, and tumor metastasis. Proc Natl Acad Sci USA 98: 3352-3357, 2001.

3. Labelle M, Begum S and Hynes RO: Direct signaling between platelets and cancer cells induces an epithelial-mesenchymal-like transition and promotes metastasis. Cancer Cell 20: 576-590, 2011.

4. Buergy D, Wenz F, Groden C and Brockmann MA: Tumor-platelet interaction in solid tumors. Int J Cancer 130: 2747-2760, 2012.

5. Rao AK and Rao DA: Platelets signal and tumors take off. Blood 120: 4667-4668, 2012.

6. Douglas VK, Tallman MS, Cripe LD and Peterson LC: Thrombopoietin administered during induction chemotherapy to patients with acute myeloid leukemia induces transient morphologic changes that may resemble chronic myeloproliferative disorders. Am J Clin Path 117: 844-850, 2002.

7. Stone RL, Nick AM, McNeish IA, et al: Paraneoplastic thrombocytosis in ovarian cancer. N Engl J Med 366: 610-618, 2012.

8. Wang-Tilz Y, Tilz C, Wang B, Tilz GP and Stefan H: Influence of lamotrigine and topiramate on MDR1 expression in difficult-to-treat temporal lobe epilepsy. Epilepsia 47: 233-239, 2006.

9. Ma S, Liu X, Geng JG and Guo SW: Increased SLIT immunoreactivity as a biomarker for recurrence in endometrial carcinoma. Am J Obstet Gynecol 202: 68 e61-e68, e11, 2010.

10. Feng W, Madajka M, Kerr BA, et al: A novel role for platelet secretion in angiogenesis: mediating bone marrow-derived cell mobilization and homing. Blood 117: 3893-3902, 2011.

11. Manetta A, Satyaswaroop PG, Hamilton T, et al: Radio imaging of human ovarian carcinoma xenograft in nude mice. Gynecol Oncol 28: 292-299, 1987.

12. Holmes CE, Levis JE and Ornstein DL: Activated platelets enhance ovarian cancer cell invasion in a cellular model of metastasis. Clin Exp Metastasis 26: 653-661, 2009.

13. Trikha M, Zhou Z, Timar J, et al: Multiple roles for platelet GPIIb/IIIa and alphavbeta3 integrins in tumor growth, angiogenesis, and metastasis. Cancer Res 62: 2824-2833, 2002.

14. Dardik R, Savion N, Kaufmann Y and Varon D: Thrombin promotes platelet-mediated melanoma cell adhesion to endothelial cells under flow conditions: role of platelet glycoproteins P-selectin and GPIIb-IIIA. Br J Cancer 77: 2069-2075, 1998.

15. Matsui H, Harada I and Sawada Y: Src, p130Cas, and Mechanotransduction in Cancer Cells. Genes Cancer 3: 394-401, 2012.

16. Nick AM, Stone RL, Armaiz-Pena G, et al: Silencing of p130cas in ovarian carcinoma: a novel mechanism for tumor cell death. J Natl Cancer Inst. 103: 1596-1612, 2011.

17. Sawada Y, Tamada M, Dubin-Thaler BJ, et al: Force sensing by mechanical extension of the Src family kinase substrate p130Cas. Cell 127: 1015-1026, 2006.

18. Zhang P, Guo A, Possemato A, et al: Identification and functional characterization of p130Cas as a substrate of protein tyrosine phosphatase nonreceptor 14. Oncogene 32: 2087-2095, 2013.

19. Chatzizacharias NA, Kouraklis GP and Theocharis SE: Clinical significance of FAK expression in human neoplasia. Histol Histopathol 23: 629-650, 2008.

20. Goel HL and Mercurio AM: VEGF targets the tumour cell. Nat Rev Cancer 13: 871-882, 2013.

21. Hanahan D and Weinberg RA: Hallmarks of cancer: the next generation. Cell 144: 646-674, 2011.

22. Prow D and Vadhan-Raj S: Thrombopoietin: biology and potential clinical applications. Oncology (Williston Park) 12: 1597-1604, 1607-1598; discussion 1611-1594, 1998. 
23. Dardik R, Kaufmann Y, Savion N, et al: Platelets mediate tumor cell adhesion to the subendothelium under flow conditions: involvement of platelet GPIIb-IIIa and tumor cell alpha(v) integrins. Int J Cancer 70: 201-207, 1997.

24. Konstantopoulos K and Thomas SN: Cancer cells in transit: the vascular interactions of tumor cells. Annu Rev Biomed Eng 11: 177-202, 2009.

25. McCarty OJ, Mousa SA, Bray PF and Konstantopoulos K: Immobilized platelets support human colon carcinoma cell tethering, rolling, and firm adhesion under dynamic flow conditions. Blood 96: 1789-1797, 2000.

26. Ay C, Dunkler D, Pirker R, et al: High D-dimer levels are associated with poor prognosis in cancer patients. Haematologica 97: 1158-1164, 2012.

27. Dirix LY, Salgado R, Weytjens R, et al: Plasma fibrin D-dimer levels correlate with tumour volume, progression rate and survival in patients with metastatic breast cancer. Br J Cancer 86: 389-395, 2002.

28. Zigler M, Kamiya T, Brantley EC, Villares GJ and Bar-Eli M: PAR-1 and thrombin: the ties that bind the microenvironment to melanoma metastasis. Cancer Res 71: 6561-6566, 2011.

29. Cowell LN, Graham JD, Bouton AH, Clarke CL and O'Neill GM: Tamoxifen treatment promotes phosphorylation of the adhesion molecules, p130Cas/BCAR1, FAK and Src, via an adhesion-dependent pathway. Oncogene 25: 7597-7607, 2006.

30. McLean GW, Carragher NO, Avizienyte E, et al: The role of focal-adhesion kinase in cancer - a new therapeutic opportunity. Nat Rev Cancer 5: 505-515, 2005.

31. Ward KK, Tancioni I, Lawson C, et al: Inhibition of focal adhesion kinase (FAK) activity prevents anchorage-independent ovarian carcinoma cell growth and tumor progression. Clin Exp Metastasis 30: 579-594, 2013.

32. Chen XL, Nam JO, Jean C, et al: VEGF-induced vascular permeability is mediated by FAK. Dev Cell. 22: 146-157, 2012.

33. Erler JT, Bennewith KL, Nicolau M, et al: Lysyl oxidase is essential for hypoxia-induced metastasis. Nature 440: 1222-1226, 2006.
34. Kirschmann DA, Seftor EA, Fong SF, et al: A molecular role for lysyl oxidase in breast cancer invasion. Cancer Res 62: 4478-4483, 2002.

35. Baker AM, Bird D, Lang G, Cox TR and Erler JT: Lysyl oxidase enzymatic function increases stiffness to drive colorectal cancer progression through FAK. Oncogene 32: 1863-1868, 2013.

36. Baker AM, Cox TR, Bird D, et al: The role of lysyl oxidase in SRC-dependent proliferation and metastasis of colorectal cancer. J Natl Cancer Inst 103: 407-424, 2011.

37. Lapointe J, Li C, Higgins JP, et al: Gene expression profiling identifies clinically relevant subtypes of prostate cancer. Proc Natl Acad Sci USA 101: 811-816, 2004.

38. Battegay EJ, Rupp J, Iruela-Arispe L, Sage EH and Pech M PDGF-BB modulates endothelial proliferation and angiogenesis in vitro via PDGF beta-receptors. J Cell Biol 125: 917-928, 1994.

39. Anglesio MS, George J, Kulbe H, et al: IL6-STAT3-HIF signaling and therapeutic response to the angiogenesis inhibitor sunitinib in ovarian clear cell cancer. Clin Cancer Res 17: 2538-2548, 2011.

40. Harker LA, Marzec UM, Kelly AB, et al: Prevention of thrombocytopenia and neutropenia in a nonhuman primate model of marrow suppressive chemotherapy by combining pegylated recombinant human megakaryocyte growth and development factor and recombinant human granulocyte colony-stimulating factor. Blood 89: 155-165, 1997.

41. Grossmann A, Lenox J, Ren HP, et al: Thrombopoietin accelerates platelet, red blood cell, and neutrophil recovery in myelosuppressed mice. Exp Hematol 24: 1238-1246, 1996.

42. Bergmeier W, Piffath CL, Goerge T, et al: The role of platelet adhesion receptor GPIbalpha far exceeds that of its main ligand, von Willebrand factor, in arterial thrombosis. Proc Natl Acad Sci USA 103: 16900-16905, 2006.

43. Cho MS, Bottsford-Miller J, Vasquez HG, et al: Platelets increase the proliferation of ovarian cancer cells. Blood 120: 4869-4872, 2012. 